\title{
BMI, waist-to-height ratio and body fat mass in older adults: results from the Pronutrisenior project
}

\author{
Flora Correia ${ }^{1,2}$, Bruno M. P. M. Oliveira ${ }^{1,3}$, Rui Poínhos ${ }^{1}$, Anzhela Sorokina ${ }^{1}$, \\ Cláudia Afonso ${ }^{1}$, Bela Franchini ${ }^{1}$, Bárbara Pereira ${ }^{1}$, Luís Fonseca ${ }^{4}$, Miguel Sousa ${ }^{4}$, \\ Ana Monteiro ${ }^{4}$ and Maria Daniel Vaz de Almeida ${ }^{1,5}$ \\ ${ }^{1}$ Faculdade de Ciências da Nutrição e Alimentação da Universidade do Porto, Porto, Portugal, \\ ${ }^{2}$ Centro Hospitalar de S. João, E. P. E., Porto, Portugal, \\ ${ }^{3}$ LIAAD - INESC TEC, Porto, Portugal, \\ ${ }^{4}$ Faculdade de Letras da Universidade do Porto, Porto, Portugal and \\ ${ }^{5}$ GreenUPorto, Porto, Portugal
}

\begin{abstract}
Near $20 \%$ of the Portuguese population is aged 65 years or above, a value similar to most developed countries. This older adult population also suffers from obesity and obesity-related pathologies. The environment encompasses a set of obesity determinants and knowing the associations between the environment and obesity may help health professionals and caregivers to provide for the older adults.

In this study, we aimed to relate anthropometric measures with socio-demographic data in older adults.

This is a cross-sectional study using data from the Pronutrisenior project, collected in Vila Nova de Gaia, Portugal. The sample consists of 456 older adults, aged 65 to 92 years without cognitive impairment. The sample consisted of older adults living at their homes. Socio-demographic, clinical, geographical, and anthropometric data was collected. The statistical analysis used IBM-SPSS-22.0 and consisted on descriptive statistics, Pearson correlations and UniANOVA. Significantly results $(p<0.05)$ are reported.

These older adults were mostly females $(54.2 \%)$ with a mean age of 73.8 years $(\mathrm{sd}=6.3)$, mean body mass index $(\mathrm{BMI})$ of $29.1 \mathrm{~kg} / \mathrm{m}^{2}$ $(\mathrm{sd}=4.8)$, mean waist-to-height ratio $(\mathrm{WHtR})$ of $0.623(\mathrm{sd}=0.073)$ and mean percentage of body fat mass $(\% \mathrm{BFM})$ of $40.7 \%(\mathrm{sd}=$ $8.9 \%)$ for females and $30.2 \%(\mathrm{sd}=8.5 \%)$ for males. BMI, WHtR and $\%$ BFM were positively correlated. In this sample of older adults, higher values of these measures were associated to being female, younger, less educated; to having articular pains and respiratory problems, and not having insomnia, hypertension, chewing problems nor hearing problems; to drink more liquids but not consuming dairy products daily; to not take nutritional supplements but to take more medicines; to be without somebody to talk to and to be more dependent; and to live in a house without stairs to climb and to live near other older adults, and in a more urbanized area with streets with steeper slopes.

In this sample of older adults, obesity is related with health characteristics and those are related with socio-demographic and geographical characteristics of the area of residence. Besides the identification of risk factors for the older adult population, this information may help designing health care policies that takes in consideration the physical and geographical characteristics of the neighbourhood of the area of residence of the older adults.
\end{abstract}

\section{Conflict of Interest}

There is no conflict of interest 